duits sur des étangs à rendement supérieur, afin que la sélection soit convenahle. Donc ıo à İ̈.ooo hedtares seront nécessaires à la production du nourrain destiné à multiplier au moins ; fois son poids. Pour avoir res 3 millions de hilogramnes de nourrains, il laut trouver 300.000 kilogrammes de feuilles capables de donmer ro fois leur poids; il suffira d'affecter $1 . \bar{j}$ oo hectares à la poute. Or, à cette heure, nous avons 20.000 hectares empoissonnés un peu régulièrcment el il ne se fait pas ro.ooo kilogrammes de feuilles sélectionnées et 15.000 de nourrains.

On voit qu'il y a de la marge pour la production de l'alevin.

Nous ne dirons rien de la pratique, du métier du producteur : c'est un autre chapitre. Nous voulions seulement exposer les avantages de l'acquéreur d'empoissonnage sélectionné et montrer que la production de cet empoissonnage dépend de l'organisation de la commande. Vous en avez déjà tiré les conclusions.

\title{
LE FÉTICHISME DE L'ÉTIQUETTE
}

\author{
Par M. Ritoul de IDROUIN de BOUVILLE
}

M. Luérituen est un émule de Bollesu : il appelle un chat un chat et souhaiterait que la mode s'en répandît (1).

Non seulement il se refuse à prendre les vessies pour des lanternes, mais il prend plaisir à les déžonller par de malicieuses piqùres.

Champion de la clarté, de l'ordre et du bon sens il ne craint pas d'émouvoir des candeurs ou de déranger des combines. Ne s'est-il pas avisé, récemment, de réclamer une rectification d'élat civil pour la Perche-Truite? plus et mieux de confester le droit des Carpes gauloises à se parer de titres inscrits au Gotha germanique?

Sur le premier chef du réquisitoire, concédons que la dénomination couramment attribuée au Microptère salmoüde induit en erreur les profanes. Ne le voient-ils pas se présentant ì la façon de l' "Amour moderne ":

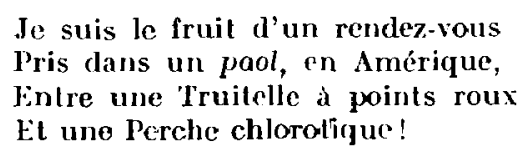

Or, il ne s'agit aucuncment d'un métis ; ce citojen des Elats-Tnis appartient à une honorable famille: celle des Centrarchides, est de bonne vie et mours et on ne lui connaît pas de liaisons, comme à la Brême, au Gardon et autres Cyprins de petite vertu. C'est lui faire tort

(1) Voir Bulletin de septombre, p. $5 \mathrm{r}$. 
que le gratilier d'un sobriquet hybride. Alors surlout que tant de bonnes gens s'ébąhiront en toute naỉveté le jour oì un Barnum leur exhibera le produit du rroisement de la Carpe avec le Lapin...

Le Hicroplère a droit à réparation, encore que Lacćpède, qui le baptisa en 180\%, hui ait trouvé, dès l'abord, un faux-air salmoîde... Mais comment l'appeler ! "Blucli-Bass ", c'est-à-dire Perche noire, comme dans son pays d'origine ? Il y aura encore des ohjoctions. Le Microptère n'est pas une Perche et il n'est pas nèzre ; sa livrée est demi-deuil ou, plus exactement, gris-verdàtre et blanc-grisâtre...

Alors usons, pour désigner la fallacieuse Porche-Truite, do sa dénomination scientifique... Ainsi se mettront a l'abri de la critique les rares amateurs de ce Poisson transatlantique.

Ceux qui ćlevent les Carpes dites de race sont, au regard, léglon à en juger par l'effectif des treize compagnies, sections ou escouades qui s'assemblent sous la bannière de l'ti. N. S. E.

Aussi ła suspicion jetée sur le blason de son cheptel ne saurait laisser indifférent ancun exploitant de nas étangs. Il s agrit de l'espèce constituant lo fond de leur peuplement. Le lièure levé par M. Lnéritren demande à être couru; la question est d'importance. Oui ou non les étiquettes des aquariums d'exposition abusent-elles les citoyens qui viennent admirer les produits de notre pisciculture et, en teste, $M$. le Président de la République ? Oui ou non, présente-t-on au peuple des Lausitz-ersatz, des Wittingau de fantaisie, des Iranconiennes sans papiers et de suspectes Bohémiennes?.

Il est patent que, depuis la guerre, il y a, dans nalre France démoratique, une remarquable floraison de Carpes aristecrates autant qu'exotiques. Il est permis d'écarquiller les yeux devant les paneartes qu'elles arhorenl, venant i la ville parader devant les citadins. A leur vue, un quidam que je connais bien ne se laissait-il pas aller à accommoder, comme suit, un couplet d'une vieille chanson de Mac-Nab sur l'expulsion des princes :

$$
\begin{aligned}
& \text { "Aischgrund" on connaît c'Poisson là ! } \\
& \text { Faut-il qu'son orgueil soye profonde } \\
& \text { Pour s'êt' fichu un nom comme ça! } \\
& \text { Peut-il pas s'app'ler comme'tout I'monde ? }
\end{aligned}
$$

Par une association d'idées similaịre, à la lecture de la dénonciation visant limposture des Carpes à sang prétendu bleu, me sont revenus à l'esprit des souvenirs récoltés an cours d'ạsez lointains voyages. Il peut y avoir profit à les évoquer pour l'appréciation du litige.

Sans doute, au premier abord, les deux anecdotes que je m'en vais narrer semblent-elles n'avoir arec la pisciculture que des rapporty plutôt vagues... Elles en ont cependant plus qu'il n'y a apparence et, tout d'abord, celui d'avoir été notées au eours de missions à l'étranger dont j'ai eu l'honneur d'être chargé par la Direction généralo des Eaux et Forèts. 
Ainsi, en Fàrier 190:, n’étant encore que Garde général, me trouvai-je à Pétersbourg - non encorc Pétrogradé - à I'occasion d'un Congrès international d'aquicullure et de pêlue.

Ce que fut l'accueil de nos amis et alliés d'alors, ceux-là seuls s'en font idée qui ont connu la Russie trariste an temps de sa splendeur.

Quelles réceptions inoubliables! Quels menus ! Et comment arrosés ?... Si communicalive était la chaleur des banquets qu'au terme de l'un d'eux, officiel, j'ai ouï, tout éberlué, S. F. le Ministre de l'Agriculture et des Domaines porter un toast à l'Eerevisse, symbole du progrès... parce qu'elle marche à reculons!

Mais parlons des crus, car cest à eux ijue jen veux venir.

A un quelconque festin. mon atlention. fut attirée par l'étiquette de la "bordelaise " posée devant noi. Somptueusement dorée, elle portait, en caractères russes, la mention " llaut-Sauternes".

Etonné, je m'enquis auprìs de " notre oncle ", c'est-à-dire du Moscovite spécialement chargé de piloter, renseigner et tenir en liesse la. délégation française. - "I'ourquoi, demandai-je, avoir supprimé ou omis la mention d'origine? Ist-re du Chatteau-Yquem on du ChàteauGuirand?"

A quoi il me fut répondu que le "Sauternes " du llacon venait de Crimée!

Et comme je réprimai insulfisamment l'expression de ma stupéfaction, on m'expliqua fort aimablement que le vignoble de Chersonèse, dont je dégustais le produit, avail été constitué au moyen de plants directement importés du canton de Langron, arrondissement de Bazas, département de lit Gironde.

La souche sautemoise élait authentique... indiscutable...

Nombre d'années après, en Juin 1925, c'est à un vieil Inspecteur, en instance de'retraite, qu'échut l'heur et l'honneur de représenter l'Administration des Eaux et Forèts au XIII Congrès international d'agriculture, à Varsovie.

Ce fut, derechef, un enchantement !

Comment louer congrument l'hospitalité polonaise, si avenante, courtoise et somptueuse? Pour lés délégués de la France surtout, que d'attentions! que de cordialité !

Les banquets se succédèrent qui sont le charme et, peut-être, lessentiel des Congrès ; car c'est là que, dûment arrosée, prend conscience et s'affirme l'humaine fraternité. Fn dépit de la confusion des langues, Babel n'est plus, alors, qu'un biblique souvenir...

Ainsi advint-il que je me retrouvai, certain jour, en présence dune " bordelaise " à splendide étiquette, rappelant la robe de la Montespan : " d'or, rebattue d'or, rebrochée d'or, surbrodée d'or ".

Cette fois, le libellé était en francais et annonçait du "Graves ". Mais quel? Haut-Brion? Picque Caillou? Rigailloux? Crorque-Michotte? 
Il me parut prudent de ne pas interroger mes hôtes. Car s'il y a des vignobles dans la Russie méridionale, en Pologne, il en va du raisin comme de la fleur d'orange place Pigalle... I en a pas beaucoup!

La chance mavait d'ailleur's placé, à table, tout à côté d'un compatriote spécialement compétent, en sa qualité de Vice-Président de la Confédération générale des vignerons. Je consultai discrètement l'excel-

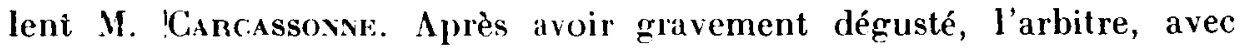
gravité, formula son verdict en ces termes : "Il y a du Graves!"

Que les pisciculteurs entendent, qui ont des oreilles pour entendre... car, pour eux, ces deux historiettes prennent tournure de paraboles.

Que sont, au fond, les ('arpes plus ou moins blasonnées de nos modernes éleveurs, contre lesquelles $M$. Lubnrikn s'en-va-t'en guerre, sinon l'équivalent ichthyotechnique du Sauternes russe ou du Graves polonais?

Si, depuis les importations réalisées de 190i à 1913 par MM. Hrrsci et Mruciniot, les nobles Poissons portant les couleurs de diverses provinces allemandes ou tchèqụes ont pratifué. exclusivement, les unions entre consanguins... alors la race est pure. Nous avons des "Wittingau " en France comme les Russes ont du "Chàteau-Yquem " en Crimée.

Qu'au contraire se soient produits des croisements, conscients ou inconscients... qu'un enfant de "Bohème " ait frayé avec une "Silésienne "... pis encore, qu'une fille de bonne maison germanique ait dérogé avec un roturier brenneux... Alors, alors, il y a de la "Lausitz " dans nos élangs comme il y a du Haut-Brion dans le "Graves " sarmate.

Voilà, comme conclusion de deux contes après boire, une morale qui étonnera, sans doute, ceux qu'elle ne contristera pas.

Ces derniers auront tort, car de grâce, qu'on ne me fasse pas dire ce que je n'ai pas dit !

Le "Haut-Sauternes " bu en 1902 m’a laissé le meilleur souvenir ; la dominante de son bouquet fleurait la vieille souche girondine. Le "Graves " dégusté en igaj ne le lui cédait guère, il s'y trouvait comme inclus, rutilant emmi d'autres, transpyrénéens, un rayon du soleil de Guyenne. Bref, dans un cas comme dans l'autre, le vin généreux, parfumé, flattait le goût et l'odorat. Mais l'étiquette était fallacieuse.

Pourquoi cette étiquette ?... Fétichisme :

Le vin de mon ami, dont Povruov chante le los, se révèle éminemment discret :

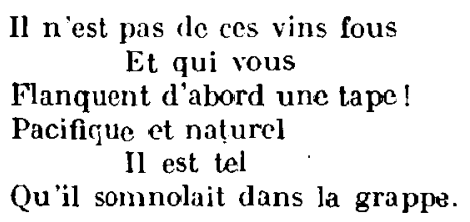

Il n arbore pas des titres ronflants... et discutables avec la prétention 
d'impressionner le palais en en mettant plein l'oil. Il se livre, confiant, à l'appréciation du connaisseur.

Les Carpes à croissance rapide qu'on obtient maintenant en France n'auraient-elles pas intérêt à s'inspirer de cette modestie, compagne habituelle du vrai mérite ?

Mais, objecteront les éleveurs de ces sujets d'élite mais de noblesse incertainẹ, quand leur qualité est indéniable, à quoi tient alors cette dernière?

La réponse tient en deux mots : " I vous! ". Car l'influence du producteur sur le produit est, en la matière qui nous occupe, sinon exclusive, du moins largement prépondérante.

Il faut, naturellement, pour avoir de bon empoissonnage, disposer d'un cheptel de belle vigueur et conformation. Peu importe que celles-ci dérivent ou non d'une illustre origine.

Mais il n'est pas suffisant d'avoir des Carpes râblées, charnues, bossues à souhait pour obtenir des feuilles susceptibles de devenir telles. Le nécessaire, l'essentiel c'est que le pisciculteur soit compétent et consciencieux. Ciompétent pour maintenir ses Poissons en condition et santé et exercer le contrôle requis sur leurs amours. Consciencieux pour ne livrer que des alevins dont il puisse garantir la bonne venue.

Ainsi les acquéreurs auront-jls satisfaction. Peu leur chant l'étiquette sous laquelle leur sont vendus des carpillons, lant qu'elle se rérère à leur ascendance. Quelle sécurité Jeur donne-t-elle autre qu'illusoire? L'assurance d'ĉtre bien servis, seule la confère la réputation du vendeur dont la capacité et la probité professionnelles sont bien établies. Cette bonne renommée leur vaut ceinture dorée.

Conclusion : ce qui fait la Carpe d'élite, c'est le pisciculteur d'élite, La qualité dı Poisson, c'est l'intégration dans son organisme, c'est l'expression ichthyobiologique des qualités intellectuelles et morales de celui qui l'élève.

A l'instar de Burros on doit poser l'éfuation : "La Carpe : c'est l'Homme!"

Qu'on se le dise !...

Foin donc des vocables avantageux, des litres exotiques, des généalogies impressionnätes... Trompe-l'neil que tout cela...

Ne soyons plus les dupes du fétichisme de l'étiquette. Que les Carpes de notre France se présentent bravement, loyalement, sous les seules couleurs de leurs éleveurs français. Jes transactions y gagneront en simplicité, clarté et loyauté ; vendeurs comme acheteurs y trouveront leur compte.

Et ainsi se trouvera enfin épargnée au jury, lors des expositions futures, la colle insidieuse posée par M. LhérITIİ, à savoir l'appréciation " des caractères distinctifs de la race " chez les Bohémicnnes limousines et les Silésiennes solognotes. 